\title{
Visualization of Music: An Aesthetic Study of the Combination of Music and Animation \\ Wen-Si OU-YANG
}

\author{
Music College, Shaanxi Normal University, No. 620, West Chang' an Avenue, Chang' an District, \\ Xi'an 710119, Shan-Xi, China \\ ouyangjusco@snnu.edu.cn
}

Keywords: Music, Animation, Vsualization, Aesthetics.

\begin{abstract}
This paper discusses the relationship between auditory sensation and visual sensation by systematically exploring traditional practical methods of music appreciation. On one hand, sound is combined with an image with the intention of more thoroughly representing the multi-dimensionality and complexity of life and the inner characters within the play, and in order to depict the profound connotations in music. On the other hand, on the aspect of music creating catharsis and drama environment, it may be complemented by images to strengthen the ability to create a sufficiently sad or joyful atmosphere, forming a unique audio-visual stereoscopic atmosphere to promote the popularity of the music.

Music is an auditory form of art which involves both sound and time. People can express their emotions, and convey and exchange ideas by using specific audio materials to create unique musical images. This practice performed by sensing, experiencing and comprehending music is known as musical appreciation. Music appreciation is very much a subjective form of aesthetic activity which occurs naturally as a result of listening to music. Moreover, it is a unique, complex and creative form of psychological activity, and is also an fundamental feature of the musical practice for mankind, as well as an organic component of our lives. Rapid advances in digital information technologies and popularity of the Internet, are inevitably bringing about a whole new revolution in musical activities that rely on auditory sensation, making it thus necessary to expand the realms of knowledge, including aesthetics, ideas and indeed everything within the field of music. An interaction between music and animation and needless to say other disciplines is unavoidable given the ever increasing speed of technological advancement, popular mass media and the diverse range of musical culture industries. Examples of this phenomenon can be seen in the wide availability of music to the public, the impact of images and videos on the distribution of music, and the overall effect of technology on music appreciation.
\end{abstract}

\section{The Accessibility of Music}

Advances in social civilization were accompanied by an increasing demand and a greater capacity for music. Instead of criticizing and maligning the 'incomprehensible' music, listeners began to understand and accept music by expanding their culture. The composition, 4'33", by avant-garde composer, John Cage, debuted in 1952. This was a work that contained no musical notes whatsoever, but represented the philosophical perspective of John Cage that music should be all about listening. He merely stepped on to the stage, picked up the baton and did not move. After a moment he turned a page and took out a handkerchief to wipe the sweat from his face. After four minutes and thirty-three seconds precisely had elapsed, (hence the title of the composition), the play was over. The audience in the concert hall was somewhat confused at the performance, or lack of it. Suddenly, someone stood up to clap, and soon after there was a whole barrage of applause pervading the auditorium. The most famous behavioral work, Cut Piece by avant-garde artist Yoko Ono debuted in 1964 at Yamaichi Concert Hall to highlight her stance on feminism that women were being treated like sexual objects. During the performance, she chose several members from the audience to step onto the stage, to cut pieces of her clothes off with scissors until she was almost naked. The play was performed again in London in 1966, causing a sensation throughout the art 
circles. In reviewing the development of art, it should be noted that whatever the style and form of the art, the audience would usually go through the phases of contempt, anger, impugnment, indifference, and finally acceptence. With the advances in social civilization, this transition has become much quicker. Music has become an indispensable part of people's lives. Information traditionally featured and conveyed by music is nowadays insufficient to meet the public's increasing demand. Mainstream music is evolving and tends to be customized to the ever-growing needs of the general public.

\section{The Distribution of Music in the Age of Image}

During the era of digital images, music no longer relied on auditory sensation only. From the late 19th century through to the early 20th century, various innovations were made in terms of metallurgy, shipbuilding, machine building, together with new methods of conveyance and telecommunication. On December 28th 1895, the first film The Arrival of the Mail Train was shown by the French Lumière brothers in Paris, highlighting the advent of a film exhibiting multi-dimensional space. In 1927, sound started to be added to films. Artists and scientists began to review the relationship between auditory sensation (time) and the visual sensation (space) from various standpoints, and since then the scope of music-based expression has been continuously developed. Music is a process that develops over time and has no intuitive stereoscopic form. It is therefore difficult for music to adequately express ideas and emotions without the use of other expressive means. What music expresses is more related to associations formed in the brain as well as the variations in imagination and psychological factors of listeners. In the context of this image age of attention-based economy, the mere auditory reproduction of music is no longer sufficient to meet the general demand of music appreciation. Instead, the active minds are constantly seeking for images matching the auditory sensation to fulfill the purpose of obtaining new artistic experience.

\section{The Impact of Technology on Music Appreciation}

Technological innovation provides a new means of music appreciation. Technological development in computers software, CGI and video apps has maximized the visualization and appealing effect of music. Music is readily available to a larger spectrum of listeners. Images, which previously only existed in our minds and imaginations can now be displayed in reality before us, and in full color. Nowadays, since being processed by computer-based digital technology, music and the related visual design works have become more desirable and capable of stimulating the sensory organs of the audience. This breaks the traditional habit of people while listening to music. Alternatively people can now enjoy and experience the emotional sensation acquired from music more freely, comfortably and directly. Walt Disney completed a grand masterpiece in 1940, called Fantasia. It was the first attempt to combine music with art and to communicate music using visual sensation (animation). In addition, this was also the earliest stereoscopic film in history. Fantasia was later reproduced in the year 2000, using the most up-to-date technologies. This film combined both traditional music with animation and was the first film to be produced using the world's most captivating movie experience, IMAX (maximum image). These two important works have made classic music 'visible' to the people, and are educational as they reveal some social phenomena by skillfully using both music and animation as the plot develops. Furthermore, these two films significantly aided adults and children alike with their comprehension and awareness for classic music. These superb technologies inevitably inject new life into music and also provide people with a spectacular visual-auditory feast. Music appreciation has evolved immensely since it was based on the aural detection of music only, to an advanced combination of auditory and visual sensations, enabling musical experience and interpretation to play a greater role in education and even in social life.

The analysis above illustrates that in the current context, music visualization conforms to the particular trends of the era, brings about new requirements in terms of music appreciation, and shares a subtle relationship with other forms of art. The high-tech transition between music and the 
dynamic sound effects of visual art can be analyzed to provide audience with altogether novel aesthetic ideas.

With the constant advances in technology, and owing to the use of novel multimedia techniques, this abstract form of non-semantic, non-formative and uncertain art is diversified, becoming more authentic in its entirety, and 'visible' to its audiences. The musical language comprises tone, interval, melody, rhythm, harmony and tone quality, which are all organized according to their particular patterns. The expression of music language is a dynamic process of musical exchange between those using this type of language. For example, composers create a musical piece to express their inner feelings, or performers express the ideological contents of the work via the medium of an instrument. Both of these examples represent different expression means in the music language and both also rely on a psychological exchange between people.

With advances in technology and social civilization, motivated to explore further development, mankind has succeeded in introducing a visual element into music language which significantly improves the expression of this form of language, and provides even more unique, vivid, direct, enriched means. Music visualization possesses two distinct forms. First, notes and lines of the music are used to evoke the images which are consistent with the desired theme of the composers. Second, associations are made in relation to the 'color' of the music and the imagination is stimulated by the plot as sounds are visualized. This paper elaborates on the latter.

The founder of the Korb Production Company, Rimantas Lukavicius, is currently among the most influential of visual art composers. In his 3-D music video I'll be gone (a House-style song played by Mario Basanov \& Vidis), an electrocardiogram (ECG) recorder was used to display sequences of logical diagrams in four windows of components of the song. The four windows constitute a flat graph of four grids. The ECG connected the locations of the four vibration points in the song by switching from one window to another, depending on the rhythm of the music. Rimantas proposes that by using the video's four-grid location, waveform fluctuations and rhythms of auditory vibration can create an extraordinary visual surround sound. Along with fluctuations in the rhythm, waveforms plotted by the recorder change into particles which subsequently scatter upwards and gradually disappear. This spectacular 3-D effect was produced after 1 minute 46 seconds and lasts until the end of the musical composition. Rimantas created a visual aesthetic picture of the music which was entirely unique, delicate and unimaginable, and which led people's visual observation and virtual imagination to a whole new 3-D space. His exclusive work competently integrates music with 3-D technology and places the importance on the consistency between aesthetics and imagination. It is like telling an incredible story which imparts a strong impression on its audience.

From a different perspective, despite enormous efforts by numerous artists, China's market for culture is still mostly dominated by music and animation originating from Japan, Europe and the U.S. In this respect, the Chinese National Academy of Arts initiated the 'Classic and Innovative Animation Projects for the Chinese Opera' in 2007. The Chinese Opera is an antiquated, unique and traditional form of music with a long history. The purpose of this project is to exhibit Chinese Opera in the form of innovative animations using digital technology. The Pioneer of Animation for Chinese Opera, Wang Xiaodong, founded the Dongding Animation Studio in Henan and many of his works, including the Chu Opera Flowery Wall, Beijing Opera Beating the Princess Jin Zhi, Huagu Opera Plea for School Fees, and the Henan Opera Seventh-Rank Officer, have all gained much attention and thus promoted Chinese operas around the world.

In terms of classical music, "the Series of Animation On Classic Music" was the first original program to be imported by the China Record Shenzhen Corporation. It is also China's first import of a program which interprets world classical music through animation. It has six series of DVD, including exquisite Vienna forest pieces, the renaissance of the piano, emotion dance, Scenery on That Side, and The People. Each of the series features its own unique artistic style and combines visual animation, enabled by advanced technology, with classical music. It has integrated all the creative ideas relating to traditional European art, sculpture, photography, and computer animation. Etiquette pictures are provided for 57 representative world-famous melodies, such as The Four Seasons by Vivaldi, Traumerei by Schuman), For Elise by Beethoven, Dance of Death by 
Saint-Saens, The Beautiful Blue Danube by Strauss, the Old Castle by Modest Petrovich Musorgsky, and Clair de Lune by Debussy. After gaining a comprehensive understanding of these works, music artists, painters and orchestras spent a further ten years selecting and composing the melodies, and exhibiting their magnificent expertise in the form of art. Elegant music can no longer be classified as incomprehensible. Instead of just watching a performance in an auditorium or on a video, the audience can now enjoy a unique visual-auditory form of art that combines music, pictures and animation. The release of this program caused a sensation across the globe within a short period of time with many countries importing the original full edition of the series, which inevitably exerted an unimaginable influence on the world.

\section{The Practical Connotation of the Visualization of Music}

From the discussion above, it is evident that the value created by musical melodies, which previously were only sensed through auditory sensation, have dramatically changed owing to the emergence of animation visual effects. Furthermore, the cultural value of music and animation has also been significantly improved. Firstly, a combination of music and visual animation enhances the efficiency of music appreciation and comprehension. Although music visualization is incapable of depicting every aspect and detail of the music, it can interpret the music more intuitively by employing the striking visual effects of animation. While providing additional means of understanding the music, animation accelerates the production and innovation of new genres of music. In today's fast pace of life, there are many factors which influence how people choose and comprehend their preferred music styles among the broad variety of music genres available. Hence the exquisite details provided by visual animation becomes an informative and intuitive factor that simplifies the selection and understanding of music. Therefore visualization is an important method for improving the efficiency of comprehending music. Secondly, The combination of music and animation unequivocally generates new aesthetic connotations and enjoyment. The time of a lack of culture has passed long before. The current social civilization has resulted in various changes in cultural ideology and certainly advanced it to a new level. Illustrating the beauty of music through visualization technology satisfies people's enhanced taste and spiritual need for the art while allowing them to be engrossed in and intoxicated by the art. Listening to a piece of music merely through auditory sensation may be somewhat lacking in respect of eliciting emotional response. Following the introduction of pictures or simply a collection of photos however, the blending of images and music can immediately and substantially expose listeners to the joys and sorrows depicted in the rhythm, and thus arouse their memories and emotions. By combining music with visual art, the superb technologies available nowadays inject a whole new life into the music, providing enormous scope for the development of music experience, music interpretation, music education and even social life. The contents and methods of traditional aesthetics have been immensely broadened, thereby exerting a profound influence on the values ideas of people, their society and life. And the promotion and awareness of this will also benefit both children and adults in the sense of education.

\section{References}

[1] William I Bauer, Musci Learning Today:Digital Pedagogy for Creating,Performing,and Responding to Music, 1 edition, Oxford Unicersity Press, New York,3/25/2014

[2] Angela Myles Beeching, Beyond Talent:Creating a Successful Career in Music, 2 edition, Oxford Unicersity Press, New York,10/5/2010

[3] Rebecca Coyle, Drawn to Sounde:Animation Flim Music and Sonicity(GENRE,MUSIC AND SOUND), Equinox Publishing, Sheffield, 6/22/2010

[4] Curtis Roads, Compsing Electronic Music:A New Aesthetic, 1 edition, Oxford Unicersity Press, New York,7/17/2015 
[5] Roger Scruton, The Aesthetics of Music,1 edition, Clarendon Press, Oxford, 7/29/1999

[6] Peter Kivy, Music,Langyage,and Cognition:And Other Essays in the Aesthetics of Music, 1 edition, Clarendon Press, Oxford,7/26/2007

[7] Andy Hamilton, Aesthetics and Music(Bloomsbury aesthetics), 1 edition, Bloomsbury Academic, New York,8/29/2007

[8] Richard Parncutt, Gary McPherson, The Science and Psychology of Muusic Performance:Strategies for Teaching and Learing,1 edition,Oxford Unicersity Press, New York, $4 / 18 / 2002$

[9] John A. Sloboda, Generative Processes in Music:The Psychology of Performance,Improvisation, and Composition, Oxford Unicersity Press, New York, 3/22/2001

[10] Helen L.Burz, Kit Marshall, Performace-Based Curriulum for Music and the Visual Arts:From Knowing to Showing(From knowing to Showing Seris), 1 edition,Corwin, California,6/10/1999

[11] Christopher Scoates, Brian Eno, Brian Eno:Visual Music, Chronicle Bools LLC, California, 9/24/2013

[12] Bruce Block, The Visual Story:Seeing the Structure of Film, TV,and New Media, 1 edition, Focal Press,New York, 4/23/2001

[13] Holly Rogers, Sounding the Gallery:Video and the Rise of Art-Music(Oxford Music/Media), 1 edition, Oxford Unicersity Press, New York, 4/10/2013 